\title{
Diagnostic Value of Napsin A in Small Biopsy of Non-Small Cell Lung Carcinoma
}

Rasha M. Samir*1, Ghada S. Osman ${ }^{2}$, Mervat M.F. El-Deftar ${ }^{3}$

${ }^{1}$ Department of Pathology, Faculty of Medicine, Aswan University, Egypt

${ }^{2}$ Department of Pathology, Faculty of Medicine, South Valley University, Egypt

${ }^{3}$ Department of Pathology, National Cancer Institute, Cairo University, Egypt

*Corresponding author: Rasha M. Samir, Mobile: (+20)01144418730, E-Mail: dr.rasha.samer@ gmail.com

\begin{abstract}
Background: Accurate sub-classification of non-small cell lung carcinoma (NSCLC) is crucial for the targeted therapy. In lung cancer most of tumors are un-respectable at diagnosis and small biopsy or cytological specimen is commonly the only available material for histopathology diagnosis. Although most of NSCLC can be diagnosed by routine stains, however diagnosis of poorly differentiated or undifferentiated tumors in small biopsy is challenging.

Objective: To evaluate the diagnostic value of Napsin A immunohistochemical (IHC) marker in NSCLC small biopsy.

Materials and Methods: Forty-six cases of primary NSCLC diagnosed by biopsy with subsequent resection were enrolled in this study. Subtyping of tumors was done according to 2015 World Health Organization criteria. IHC expression of Napsin A was assessed for all the cases. For large cell carcinoma (LCC) cases, Thyroid Transcription Factor 1 (TTF-1) and P63 expression were also tested. Markers expression was correlated with the histological diagnosis of the tumor. Sensitivity, specificity, positive, negative predictive values, and total accuracy of the markers were calculated.
\end{abstract}

Result: In NSCLC cases, the diagnostic sensitivity and specificity of Napsin A were $85.2 \%$ and $75 \%$ (respectively) with a PPV of $85.2 \%$, NPV of $75.0 \%$ and $81.4 \%$ accuracy. In LCC cases TTF1 sensitivity was $57.1 \%$ and specificity 100\%, PPV 100\%, NPV 14.3\%, accuracy 60.0\%. In LCC P63 marker had a diagnostic sensitivity, specificity, PPV, NPV and accuracy of 100\%, 85.7\%, and 33.3\%, 100.0\% and 86.7\% respectively. Expression of both Napsin A and TTF 1 in LCC cases raised the accuracy to $86.7 \%$.

Conclusions: In small biopsy specimens Napsin A can subtype NSCLC with high sensitivity and moderate specificity. Additions of TTF-1/p63 IHC markers can further enhance the accuracy.

Keywords: Non-small cell lung carcinoma (NSCLC), large cell carcinoma (LCC).

\section{INTRODUCTION}

Globally, lung cancer is the most common malignancy with the highest percentage of cancer related deaths. According to the latest WHO data published in 2017 Lung Cancers Deaths in Egypt reached $0.95 \%$ of total deaths. The adjusted death rate is 7.50 per 100,000 of population. Egypt ranks 116 in the world. Around 58\% of all lung cancers occur in developing countries. The age standardized rate (ASR) of incidence and mortality is higher in developed countries than less developed ones. It has a very low five-year survival rate (17.8\%) compared to other leading cancers ${ }^{(\mathbf{1}, \mathbf{2})}$.

Majority of primary lung cancer cases (99\%) are carcinomas. Primary lung carcinomas have been classified into small cell lung carcinoma (SCLC, 20\%) and non-small cell lung carcinoma. In lung cancer only approximately one-fourth of cases are surgically treated as most of tumors are unrespectable at diagnosis. Small biopsy or cytological specimen is commonly the only available material for histopathology diagnosis. When the amount of tumor is limited or the morphology is unclear immunohistochemical (IHC) stains may aid in diagnosis and precise subtyping of lung carcinoma cases ${ }^{(3)}$. Traditionally, histopathologic differential diagnosis of lung carcinoma was between SCLC and NSCLC, as sub-typing of NSCLC had not been shown to predict differences in patient outcomes. Recent advances in molecular biology have led to an increase in target-specific chemotherapies that require the subcategorization of NSCLCs. Poorly differentiated NSCLC was considered as large cell carcinoma but recently has become adenocarcinoma solid pattern (ADC-S) or non-keratinizing squamous cell carcinoma (NKSQCC) while non-small cell lung cancer with negative immune markers which cannot be categorized as ADC-S or NKSQCC named large cell carcinoma with null immunohistochemical features (LCC-N) ${ }^{(4)}$.

Thyroid Transcription Factor 1 (TTF-1) and Napsin A are both stains that have been proven to stain a majority of lung ADCs. The IHC detection of them in specimens should be routinely performed for diagnostic and prognostic significance ${ }^{(4)}$. TTF-1 is a nuclear transcript factor that is expressed in epithelial cells of the lung and thyroid. In the lung, it regulates the expression of genes involved in production of surfactant. The sensitivity and specificity of TTF-1 in the identification of lung origin vary, and range from $75 \%$ to over $95 \%{ }^{(5)}$. However, TTF-1 is also immunoreactive in several other tumors, such as thyroid neoplasms, breast adenocarcinoma, gastrointestinal carcinomas, small cell lung carcinoma (SCLC), and carcinoid, and possibly but controversially primary lung squamous cell carcinoma ${ }^{(6,7)}$. 
Napsin A (Novel Aspartic Proteinase of the PepSIN Family) is a cytoplasmic marker identified in type II pneumocytes, and alveolar macrophages and renal tubules. Functionally, Napsin A is an aspartic protease involved in the posttranslational modification of surfactant protein B (SP-B) in type II pneumocytes. Napsin A expression has been shown to be transcriptionally regulated by TTF- ${ }^{(5)}$.

Previous studies using surgical resected specimens indicated that Napsin A has a better sensitivity and specificity than TTF-1 in well to moderately differentiated lung ADCs. Therefore, it has been used with TTF-1 together in the differential diagnosis of lung adenocarcinomas ${ }^{(6,8)}$.

Although there is variability in the sensitivity and specificity of immunohistochemical markers in SqCC and ADC of the lung, a simple panel of four to five immunohistochemical stains can identify the tumor type with a high degree of confidence ${ }^{(9)}$.

The aim of our study was to evaluate the utility of Napsin A IHC marker in diagnosis of NSCLC biopsy samples, and to correlate its result whenever possible with that of P63 \& TTF1.

\section{MATERIAL AND METHODS:}

Forty-six cases of primary NSCLC diagnosed at Pathology Department of National Cancer Institute, Cairo University were enrolled in this retrospective study. All patients were diagnosed by trans-bronchial biopsy between January 2013 and December 2016 with subsequent resection. All relevant clinical and pathological data were obtained from hospital archives.

\section{Ethical statement:}

The study was approved by Ethics Institutional Review Board Committee of National Cancer Institute. Every patient signed an informed written consent for acceptance of participation.

\section{Histopathological evaluation:}

Hematoxylin and Eosin (H \& E) stained slides from both biopsy and resection specimens were examined to revise tumor histology. Tumors were classified into SCC if they showed intercellular bridges or keratinization, $\mathrm{AD}$ if they showed glandular formation or mucin production, adeno-squamous if they showed a mixture of SCC and AD features, and LCC if lacked glandular or squamous differentiation ${ }^{(\mathbf{1 0})}$.

\section{Immunohistochemistry (IHC):}

Napsin A protein expression was tested in all studied cases. In LCC p63 and TTF-1 expression were tested in addition. IHC was performed; all antibodies and solutions are used in the routine diagnostic procedures at the Department of Pathology. Representative $4-\mu \mathrm{m}$ sections from formalin fixed paraffin embedded blocks were cut. All slides were stained using automated immune-stainer (Ventana- biotech, Tucson, AZ) using commercially available monoclonal antibodies (Table
1). Appropriate positive and negative control was used in each run.

\section{IHC stain interpretation:}

A case was considered to be positive if greater than $5 \%$ of tumor cells with an appropriate staining pattern were identified, otherwise the case was considered to be negative. In terms of specific staining patterns, coarse granular cytoplasmic staining was considered positive for Napsin A (11). Nuclear staining was considered positive for TTF-1 and P63 ${ }^{(5)}$. Care was taken not to interpret entrapped normal lung bronchial epithelium or pulmonary macrophages as a positive staining.

Table (1): Antibodies used in study

\begin{tabular}{|l|c|c|c|c|c|}
\hline Protein & CLONE & Source & Dilution & $\begin{array}{c}\text { Protein } \\
\text { visualization }\end{array}$ & $\begin{array}{c}\text { Positive } \\
\text { control }\end{array}$ \\
\hline $\begin{array}{l}\text { Napsin } \\
\text { A }\end{array}$ & IP64 & Ventana & $1: 100$ & Cytoplasmic & $\begin{array}{c}\text { Breast } \\
\text { cancer }\end{array}$ \\
\hline TTF1 & $8 \mathrm{G} 7 \mathrm{G} 3 / 1$ & Ventana & prediluted & Nuclear & $\begin{array}{c}\text { Breast } \\
\text { Cancer }\end{array}$ \\
\hline P63 & $4 \mathrm{~A} 4$ & Ventana & $1: 400$ & Nuclear & $\begin{array}{c}\text { Colon } \\
\text { cancer, } \\
\text { tonsil }\end{array}$ \\
\hline
\end{tabular}

\section{Statistical analysis}

Statistical analysis was done using IBM@ SPSS $\odot$ Statistics version 22 (IBM $\odot$ Corp., Armonk, NY, USA). Numerical data were expressed as mean and standard deviation and range as appropriate. Qualitative data were expressed as frequency and percentage. Evaluation of diagnostic value of the genes was done by calculating sensitivity, specificity, positive predictive values (PPV), negative predictive value (NPV) and accuracy against gold standard by comparing results of immunostaining of biopsy with the histopathology of resected specimen.

\section{RESULTS}

\section{Clinic-pathological data:}

Out of 46 cases of primary NSCLC included in this study $37(80.4 \%)$ were males and $9(19.6 \%)$ were females. The mean age at time of surgery was $63.8 \pm$ 12.5 years. Examination of $\mathrm{H} \& \mathrm{E}$-stained sections from biopsy specimens included revealed that there were 15 (32.61\%) SCC, $13(28.3 \%)$ ADC, 1 (2.3\%) adenosquamous, and 17 (36.9\%) LCC. (Table 3). Regarding histological grade, 14 tumors were grade II $(30.4 \%)$, and $32(69.6 \%)$ were grade III.

\section{IHC expression of NAP A marker:}

In biopsy-stained slides $27 \quad(58.7 \%)$ tumors exhibited positive Napsin A expression. All ADC cases (13), 4 (14.8\%) SCC (showed granular cytoplasmic reactivity) and $10(58.8 \%)$ LCC were positive for Napsin A expression (Figure 1). Sections examined revealed that $\mathrm{LCC}$ cases were $14 \mathrm{ADC}, 1 \mathrm{SCC}$ and 2 null. Thus Napsin A had a sensitivity of $85.2 \%$, specificity of $75.0 \%$, PPV of $85.2 \%$, NPV of $75.0 \%$, and accuracy of $81.4 \%$. 


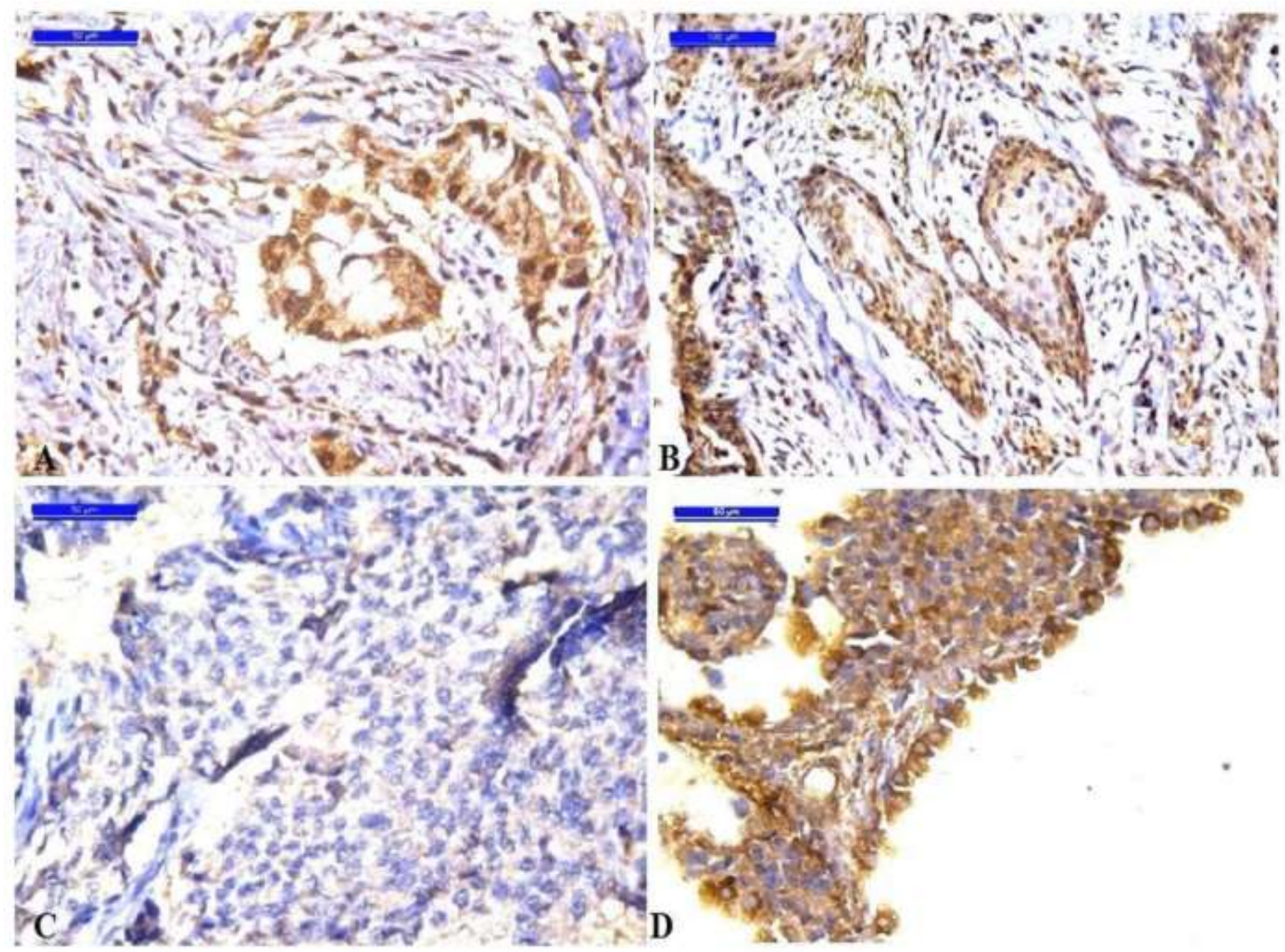

Figure (1): Nap A immunostaining in NSCLC transbronchial biopsy. Positive granular cytoplasmic stain in adenocarcinoma (A, x200) and squamous cell carcinoma (B, x100). Undifferentiated cell carcinoma negative for Nap A expression (C,x 200). Alveolar pneumocytes showed positive NAP A expression, internal control ( D, x200).

\section{IHC expression of TTF1 \& P63 in undifferentiated} group:

For TTF1, 8 out of 14 of poorly differentiated adenocarcinoma $(57.1 \%)$ cases were positive giving a sensitivity of $57.1 \%$.

On using both markers (Napsin A and TTF1), the total accuracy of diagnosis of poorly differentiated adenocarcinoma rose from $81.4 \%$ to $86.7 \%$ denoting better accuracy than with single marker use. p63 marker was positive in $2 / 14(14.3 \%)$ cases of poorly differentiated adenocarcinoma and positive in the single poorly differentiated SCC case giving sensitivity and specificity of $100 \%$ and $85.7 \%$ respectively with accuracy $86.7 \%$.

Table (2): The diagnostic value of NAP A, TTF1 and P63 IHC markers in LCC cases is presented

\begin{tabular}{|l|c|c|c|c|c|c|}
\hline \multicolumn{1}{|c|}{ Marker } & $\begin{array}{c}\text { Adenocarcinoma } \\
\text { N.14(93.3\%) }\end{array}$ & $\begin{array}{c}\text { Squamous } \\
\text { N.1 (6.7\%) }\end{array}$ & Sensitivity & Specificity & PPV & NPV \\
\hline Napsin A & $\begin{array}{c}11(10)(78.6 \%) \\
71.4 \%\end{array}$ & $0(0 \%)$ & $\begin{array}{c}78.6 \%, \\
71.4 \%\end{array}$ & $100 \% .0$ & $100 \%$ & $25.0 \%$, \\
\hline TTF1 & $8(57.1 \%)$ & $0(0 \%)$ & $57.1 \%$ & $100 \%$ & $100 \%$ & $14.3 \%$, \\
\hline P63 & $2(14.3 \%)$ & $1(100 \%)$ & $100 \%$ & $85.7 \%$, & $33.3 \%$, & $100 \%$ \\
\hline
\end{tabular}




\section{DISCUSSION}

Lung adenocarcinoma (ADC) and squamous cell carcinoma (SCC) are the main branch point in the category of non-small cell carcinoma (NSCLC). Accurate diagnosis distinguishing between these two lung cancer types has clinical significance for target therapy ${ }^{(\mathbf{1 2})}$.

When diagnosing ADC and SCC in small biopsy, cytomorphologic accuracy is reported to be $80 \%$ and $87 \%$, respectively. Inaccurate histologic typing may result from degenerative changes in ADC (such as coagulative necrosis) giving a pseudo-keratinized appearance for the tumor. Likewise, SCC cells can undergo vacuolar degeneration leading to false diagnosis of ADC. Therefore the use of IHC markers in addition to routine stains is recommended for NSCLC to enhance the diagnostic accuracy that directly affects patient management ${ }^{(\mathbf{1 3})}$.

In this study, we focused on the evaluation of the diagnostic utility of Napsin A IHC marker in the subclassification of NSCLC. The choice of antibody clone and the cut off value for positive staining are of great importance for the evaluation of IHC stains. In the literature for Napsin A the cutoff value ranged from 1\% to $10 \%{ }^{(6,10)}$. In our study we used $5 \%$ as cut off value for Napsin A.

Napsin A reactivity was detected in $23 / 27$ (all ADC + 4 Scc) of ADC denoting a sensitivity of $85.2 \%$. Our results are concordant to those reported by Whithaus et al. ${ }^{(11)}$, Turner et al. ${ }^{(14)}$ and Kim et al. (15). Authors demonstrated a sensitivity of $83 \%, 87 \%$ and $83 \%$ for Napsin A, respectively. While, Grzegorz et al. ${ }^{(5)}$, Ezzat and Tahoun ${ }^{(10)}$ and Nishino et al. ${ }^{(13)}$ showed a higher sensitivity $(89.3 \%, 92 \%$ and $94 \%$ respectively). These discrepancies between our results and others could be attributed to differences in a number of cases, the use of different clones of antibodies, and different methods of staining. Tissue fixation procedures before processing also have a significant impact on IHC expression results.

In this study, 4/16 cases of SCC showed positive granular cytoplasmic staining for Napsin A. Thus the NPV of Napsin A (specificity) was 75\%.Our results were nearly similar to those reported by Fatima and colleagues ${ }^{(17)}$ as they reported a specificity of $74 \%$. While it is lower than that recorded by Grzegorz et al. ${ }^{(5)}$, Ao et al. ${ }^{(8)}$, Ezzat and Tahoun ${ }^{(10)}$ and Nishino et al. ${ }^{(13)}$, who showed a specificity of $97 \%, 100 \%, 100 \%$ and $90 \%$ respectively. The reason for such discrepancy could be due to using a lower specificity clone of Napsin A, while the higher specificity clones are showing only focal or weak reactivity.

Our result revealed reactivity for TTF-1 in $57.1 \%$ cases of poorly differentiated ADC (8/14 cases) while none of SCC cases exhibited positive expression (NPV $100 \%$ ). This is in agreement with Kim et al. ${ }^{(15)}$ that revealed $56.6 \%$ (30/53) sensitivity for TTF-1. Siddiqui et al. ${ }^{(16)}$ reported that TTF-1 showed positive reaction in $73 \%$ of ADCs and none of the SCC specimens. The disagreement between our and their results could be due to the difference in the sample size, and tumor heterogeneity in IHC expression specifically in a small sized biopsy.

The combination of Napsin A and TTF-1 raised the sensitivity of detection of poorly differentiated ADC to $85.7 \%$ with total accuracy of $86.7 \%$. This is agreeing with Grzegorz and Colleague (5), while, Fatima et al. ${ }^{(17)}$ achieved $74 \%$ accuracy.

Regarding SCC, P63 is the commonly used as diagnostic marker. P63 can be detected in benign bronchial stem cells and in neoplastic cells with evidence of squamous differentiation ${ }^{(5)}$.

In our study $2 / 14(14.3 \%)$ of poorly differentiated ADC and the single SCC were positive for P63 with a sensitivity, specificity and accuracy of $100 \%, 85.7 \%$ and $86.7 \%$ respectively. Our result is near to that recorded by Whithausk et al. ${ }^{(11)}$ who revealed a p63 sensitivity of $95 \%$, and specificity of $86 \%$. However Grzegorz et al. (5) revealed that P63 showed a sensitivity and specificity of $91.7 \%$ and $78.3 \%$ respectively. Researchers observed that p63 usually shows weak and focal expression in ADC in contrast to strong and diffuse staining seen in SCC. These observations were explained by the possibility of glandular differentiation in $\mathrm{SCC}{ }^{(5)}$.

\section{CONCLUSION}

Napsin A can subtype NSCLC small biopsy specimens with high sensitivity and moderate specificity. Additional stains (TTF-1/p63) may be necessary for precise subtyping.

\section{REFERENCE}

1. Cheng T, Cramb S, Baade $P$ et al. (2016): The International Epidemiology of Lung Cancer: Latest Trends, Disparities, and Tumor Characteristics. Journal of Thoracic Oncology, 11 (10): 1653-1671.

2. Siegel R, Ma J, Zou Z et al. (2016): Cancer statistics. CA Cancer J Clin., 64: 9-29.

3. Shivani R, Lin F, Gui L et al. (2017): Application of Immunohistochemistry in Undifferentiated Neoplasms. A Practical Approach, 141 (8): 1014-1032.

4. Ma Y, Fan M, Dia L et al. (2015): The expression of TTF-1 and Napsin A in early-stage lung adenocarcinoma correlates with the results of surgical treatment. Tumour Biol., 36: 8085-92.

5. Grzegorz T, Zhang L, Wang Y et al. (2015): Utility of five commonly used immunohistochemical markers TTF1, Napsin A, CK7, CK5/6 and P63 in primary and metastatic adenocarcinoma and squamous cell carcinoma of the lung: a retrospective study of 246 fine needle aspiration cases. Clin Transl Med., 4: 16-25.

6. Ferlay J, Soerjomataram I, Dikshit $\mathrm{R}$ et al. (2015): Cancer incidence and mortality worldwide: Sources, 
methods and major patterns in GLOBOCAN. Int J Cancer, 136: 359-386.

7. Pelosi G, Fraggetta F, Pasini F et al. (2001): Immunoreactivity for TTF1 in stage 1non-small cell carcinoma of the lung. Am J Surg Pathol., 25: 363-72.

8. Ao M, Zhang H, Sakowski L et al. (2014): The utility of a novel triple marker (combination of TTF1, napsin A, and p40) in the sub classification of non-small cell lung cancer. Hum Pathol., 45: 926-934.

9. Carney J, Kraynie A, Roggli V (2015): Immunostaining in Lung Cancer for the Clinician. Commonly Used Markers for Differentiating Primary and Metastatic Pulmonary Tumors. Annals of the American Thoracic Society, 12: 3-9.

10.Ezzat N, Tahoun N (2016): The role of Napsin-A and Desmocollin-3 in classifying poorly differentiating nonsmall cell lung carcinoma. Journal of the Egyptian National Cancer Institute, 28 (1): 13-22.

11. Whithaus K, Fukuoka J, Prihoda T et al. (2012): Evaluation of napsin A, cytokeratin 5/6, p63, and thyroid transcription factor 1 in adenocarcinoma versus squamous cell carcinoma of the lung. Arch Pathol Lab Med., 136 (2): 155-62.

12.LU X, Chen X, Zou Y et al. (2017): Eight potential biomarkers for distinguishing between lung adenocarcinoma and squamous cell carcinoma. Oncotarget, 8: 71759-71771.

13. Nishino M, Hoang M, Pelle P et al. (2016): Napsin A/p40 antibody cocktail for subtyping non-small cell lung carcinoma on cytology and small biopsy specimens. Cancer Cytopathol., 124 (7): 472-84.

14. Turner B, Cagle P, Sainz I et al. (2012): Napsin A, a new marker for lung adenocarcinoma, is complementary and more sensitive and specific than thyroid transcription factor 1 in the differential diagnosis of primary pulmonary carcinoma: evaluation of 1674 cases by tissue microarray. Arch Pathol Lab Med., 136: 163-171

15.Kim M, Shin H, Shin K et al. (2013): Best immunohistochemical panel in distinguishing adenocarcinoma from squamous cell carcinoma of lung: tissue microarray assay in resected lung cancer specimens. Ann Diagn Pathol., 17: 85-90.

16. Siddiqui M (2012): TTF 1 and Napsin A double staining in diagnosing lung adenocarcinoma. J Cytol Histol., 3: 103-109.

17. Fatima N, Cohen C, Lawson D et al. (2011): TTF-1 and Napsin A double stain: a useful marker for diagnosing lung adenocarcinoma on fine-needle aspiration cell blocks. Cancer Cytopathol., 119: 127-133. 